\title{
Quantification of the Relationship between Sea Surface Roughness and the Size of the Glistening Zone for GNSS-R
}

\author{
Qingyun Yan, Student Member, IEEE, Weimin Huang, Senior Member, IEEE, and Giuseppe Foti
}

\begin{abstract}
A formulation of the relationship between seasurface roughness and extension of the glistening zone (GZ) of a Global Navigation Satellite System Reflectometry (GNSS-R) system is presented. First, an analytical expression of the link between GZ area, viewing geometry and surface mean square slope (MSS) is derived. Then, a strategy for retrieval of surface roughness from the delay-Doppler map (DDM) is illustrated, including details of data pre-processing, quality control and GZ area estimation from the DDM. Next, an example of application of the proposed approach to spaceborne GNSS-R remote sensing is provided, using DDMs from the TechDemoSat-1 mission. The algorithm is first calibrated using collocated in-situ roughness estimates using datasets from the National Data Buoy Center (NDBC), its retrieval performance is then assessed, and some of the limitations of the suggested technique are discussed. Overall, good correlation is found between buoy-derived MSS and estimates obtained using the proposed strategy $(r=0.73)$.
\end{abstract}

Index Terms-Delay-Doppler map (DDM), Global Navigation Satellite System-Reflectometry (GNSS-R), sea surface roughness, mean square slope (MSS), glistening zone (GZ).

\section{INTRODUCTION}

Global Navigation Satellite System Reflectometry (GNSS$\mathrm{R})$ is by now a well-established remote sensing technique, which has proven to be extremely successful in sensing ocean surface roughness and near-surface wind conditions [1]-[12]. Retrieval strategies employed so far broadly belong to three categories: a) associating an observable extracted from the delay-Doppler map (DDM) to surface roughness or nearsurface wind [1]-[6]; 2) fitting a theoretical model to measured DDMs [7]-[11]; and c) retrieving the surface scattering coefficient through deconvolution of the DDM [12]. However, this last approach is of limited application because of the difficulty associated with DDM deconvolution, along with the ambiguity linked to DD-to-space mapping [8]. Other promising applications of GNSS-R include soil moisture monitoring [13], [14], oil slick detection [15], [16], tsunami early warning [17], [18] and sea ice detection [19], [20], [21].

The glistening zone (GZ) of a GNSS-R system, also known as effective scattering region, is defined as the area delimited

Manuscript received August 21, 2017; revised October 8, 2017; accepted December 9, 2017. This work was supported by the Natural Sciences and Engineering Research Council of Canada Discovery Grants to W. Huang (NSERC RGPIN-2017-04508 and RGPAS-2017-507962).

Q. Yan and W. Huang are with the Faculty of Engineering and Applied Science, Memorial University, St. John's, NL A1B 3X5, Canada (e-mail: qy2543@mun.ca; weimin@mun.ca).

G. Foti is with the National Oceanography Centre, Southampton, SO14 3ZH, U.K. (e-mail: g.foti@noc.ac.uk). by the scattering coefficient being equal to a percentage of its maximum, which occurs at the specular point (SP) [22]. It is well known that the size of GZ depends on the ocean surface roughness [23], [24]. However, to the authors' knowledge, the relationship between extension of the GZ and surface roughness has yet to be formulated analytically, potentially offering the basis for a novel strategy for the retrieval of surface parameters. Ocean roughness is typically described using statistical properties such as mean square slope (MSS), defined as the variance of surface slope. Although it has been found that GNSS-R signals have a tighter physical link to MSS than to wind speed [11], it has been demonstrated that realistic estimates of near-surface winds can be derived from roughness using (for example) empirical models [25].

In this study, a theoretical formulation of the link between GZ area and surface roughness is first developed based on the definition of GZ in terms of scattering coefficient, which is known to depend on the probability density function (PDF) of surface slope [23]. Assuming normally distributed surface slope, a GZ-MSS relationship is derived by taking into account only slopes of favourable orientation i.e. providing reflections towards the receiver. To first order, this relationship is found to be dependent on incidence angle and distance of the corresponding surface element to the SP. This approach is then tested and its validity assessed using roughness estimates based on measurements from the TechDemoSat-1 (TDS-1) mission, ground-truthed with collocated buoy-derived surface roughness, from data of the National Data Buoy Center (NDBC). Although the proposed strategy has been successfully validated using spaceborne data, several limiting factors still exist, and more representative estimates are likely to be obtained using airborne sensors.

This letter is organized as follows: Section II gives an overview of the definition of glistening zone and discusses its mathematical formulation. Section III introduces an approximation technique, based on which a GZ-MSS relationship is later expressed. Section IV focuses on experimental validation of this model using TDS-1 measurements collocated with NDBC buoy data, and Section V gives the conclusions.

\section{Definition of Glistening Zone}

The glistening zone is defined as the region characterized by the scattering coefficient $\left(\sigma^{0}\right)$ being greater than a certain threshold (e.g. 1/e in [22], normalized to the specular point). It is known that $\sigma^{0}$ depends on the distribution of surface slope 
associated to reflections falling within the field of view of the receiver $(\mathrm{Rx})$. It has been found that $\sigma^{0}$ has a first-order link to slope distribution as the other terms vary more slowly over the glistening zone [23]. Although more realistic models of the sea-surface exist, the assumption of a zero-mean isotropic Gaussian distribution of surface slope has been widely adopted in previous GNSS-R studies (e.g., [7], [9]-[11], [17] and [26]), i.e.:

$$
P D F(s)=\frac{1}{2 \pi \cdot M S S} e^{\left(-\frac{s^{2}}{2 \cdot M S S}\right)},
$$

where

$$
s=\tan \beta
$$

is the surface slope enabling reflection of the incoming signal to the $\mathrm{Rx}$, and $\beta$ is defined as the angle between the local bisector vector (BV) and the local surface normal ( $z$-axis, using the reference frame illustrated in Fig. 1).

The BV can be expressed as:

$$
\mathrm{BV}=\left(\vec{R} /|\vec{R}|-\overrightarrow{R_{0}} /\left|\overrightarrow{R_{0}}\right|\right),
$$

where $\vec{R}$ and $\overrightarrow{R_{0}}$ are the vectors associated to the scattered and incident wave, respectively. Here, the sea-surface is assumed to be planar, as the effect of the curvature of the Earth has been shown to be negligible (e.g. [26], [27]).

With reference to Fig. 1 illustrating the geometry of the problem using a reference frame centred at the specular point, we consider $\overrightarrow{R_{0}}$ and $\vec{R}$ of a certain surface element having coordinates $(L \cos \varphi, L \sin \varphi, 0)$, and we find:

$$
\begin{aligned}
& \vec{R}_{0}=\left(L \cos \varphi+R_{t} \sin \theta, L \sin \varphi,-R_{t} \cos \theta\right), \\
& \vec{R}=\left(R_{r} \sin \theta-L \cos \varphi,-L \sin \varphi, R_{r} \cos \theta\right),
\end{aligned}
$$

where $L$ is the distance from the surface element to SP, $\varphi$ represents the azimuth angle of the surface element, $\theta$ denotes the incidence angle at $\mathrm{SP}$, and $R_{r}$ and $R_{t}$ are the distances from SP to Rx and to transmitter (Tx), respectively.

Here, $R_{r} \approx H_{r} / \cos \theta$ and $R_{t} \approx H_{t} / \cos \theta$, with $H_{r}=$ $635 \mathrm{~km}$ and $H_{t}=20200 \mathrm{~km}$ being the altitudes of TDS-1 receiver and of the GPS transmitter. Here, we intend to express $s$ as a function of distance and azimuth angle of the scattering element relative to the SP, and of the incidence angle at the SP.

As $\theta$ is independent of $L$, and simulations have shown that $s$ only weakly depends on $\varphi$, the dependence can be approximated as the product of two separate functions of $\theta$ and $L$, i.e.:

$$
s(\theta, L)=f(\theta) \cdot g(L)
$$

Following Eq. (1), the area of GZ can thus be written as

$$
G Z=\iint_{P D F_{n o r m}(s)>a} d A,
$$

where the normalized $P D F_{n o r m}(s)=e^{\left(-\frac{s^{2}}{2 \cdot M S S}\right)}$ (note that, $s=0$ at SP), $a$ denotes an arbitrary threshold from 0 to 1 , and $d A$ represents the surface element of the scattering area

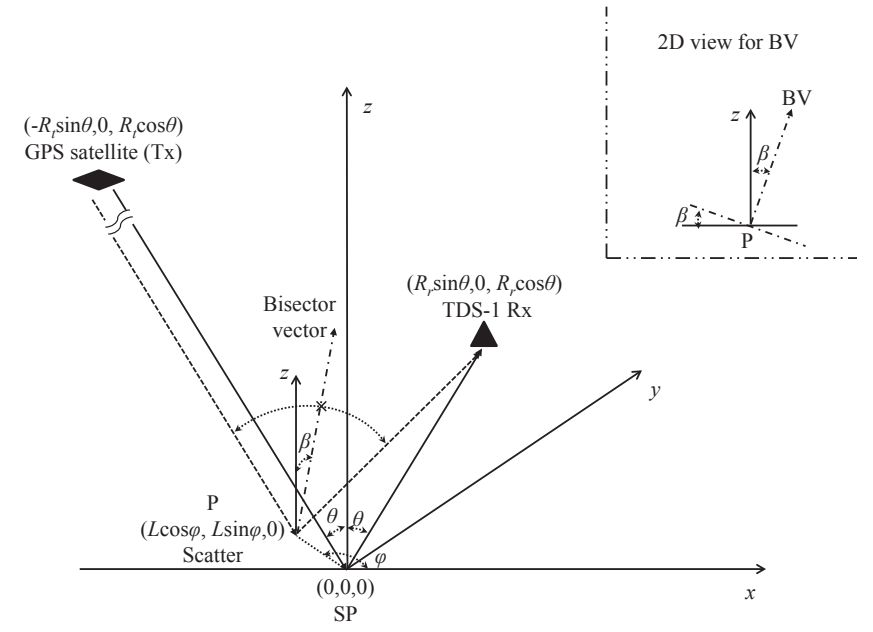

Fig. 1. Illustration of the geometry of a spaceborne GNSS-R system, using a reference frame centred at the specular point (SP). Incidence plane and local bisector vector $\mathrm{BV}$ are also indicated.

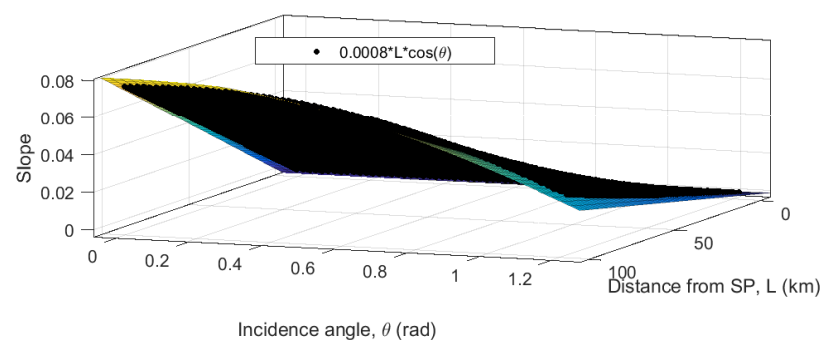

Fig. 2. Relationship between surface slope, distance from SP and incidence angle. Black dots and surface represent results obtained using the approximated function (8) and analytical formulation, respectively.

A. Rearranging Eq. (1) and substituting in Eq. (6) we find

$$
\begin{aligned}
G Z & =\iint_{s^{2}<-2 \ln (a) \cdot M S S} d A \\
& =\iint_{|g(L)|<\sqrt{-2 \ln (a) \cdot M S S} /|f(\theta)|} d A,
\end{aligned}
$$

which shows that, given a scattering coefficient threshold, the spatial extension of the GZ can be expressed in terms of incidence angle $\theta$ and surface MSS.

\section{DETERMINATION OF GZ-MSS RELATIONSHIP}

\section{A. Approximation of $s$ and Relationship between GZ and MSS}

Surface slopes enabling reflections towards the receiver can be expressed as a function of incidence angle and distance of the scattering element to the specular point. Based on the model presented above (see Eq. (5)), we assume that $f(\theta)=$ $\cos \theta$ and that the dependence on $L$ is linear i.e.

$$
s=k L \cos \theta
$$

Given TDS-1 altitude, applying least-squares fitting we find that $k_{T D S-1-S I M}=0.0008$, see Fig. 2. Clearly, this process can be extended to other low-earth-orbit GNSS-R systems using the corresponding mission parameters. 
Transforming $d A$ into $L d L d \varphi$ and arbitrarily setting $a=$ 0.1 to preserve a sufficient number of scattering elements, we find

$$
G Z=2 \pi \int_{0}^{\frac{\sqrt{4.6 M S S}}{0.0008 \cos \theta}} L d L .
$$

Or, more generally,

$$
M S S=m \cdot \cos ^{2} \theta \cdot G Z,
$$

where $m_{T D S-1-S I M}=4.4 \cdot 10^{-8}$ for simulated TDS- 1 data.

The expression obtained shows that, given the altitudes of GNSS transmitter and receiver, estimates of surface roughness MSS can be retrieved using the spatial extension of the GZ, along with incidence angle $\theta$.

\section{B. Estimation of $G Z$ Area from DDM}

Conventional spatial DD-to-space mapping methods merely rely on geometry, i.e. the surface area is estimated based on the intersection of the corresponding iso-delay and isoDoppler lines [2]. However, the concept of "effective spatial resolution" is proposed in [24], which integrates the effect of geometry and of varying scattered power across the GZ, together with the spreading effect of the Woodward ambiguity function. Additionally, this study presents a method for quick estimation of the effective spatial resolution of a certain DD interval by using a compact polynomial approximation (see [24] for details), the input parameters being incidence angle, Rx altitude and DD range.

Here, to estimate the extension of the GZ from the DDM, we adopt the technique presented in [24], evaluate the scattering area (defined as the square of the spatial resolution in [2]) corresponding to each Delay-Doppler (DD) pixel, and then obtain a total GZ area estimate by summing the individual pixel contributions.

The area of each pixel can generally be obtained by differentiating the areas of two DD intervals. Fig. 3 illustrates an example of this method using DD intervals having different resolutions. We consider a first DD interval A ([0 0.25$]$ chip/[-500 500] Hz, blue dashed rectangle) having resolution $L_{A}$, and a second larger one B ([ [ 0.25$]$ chip/[-1000 1000] $\mathrm{Hz}$, red solid rectangle) having resolution $L_{B}$. Because of symmetry, we find that DD pixels $C_{1}$ ([0 0.25$]$ chip/[-1000 -

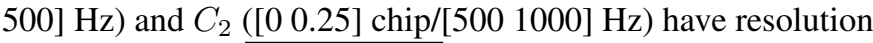
$L_{C_{1}}=L_{C_{2}}=\sqrt{\left(L_{B}^{2}-L_{A}^{2}\right) / 2}$.

In summary, the scattering areas of different DD intervals are calculated first, using the technique of "effective spatial resolution". Next, the area of each DD pixel can be derived through differentiating the areas of associated DD intervals. Last, the total GZ area can be estimated by summing the area of each DD pixel.

\section{EXPERIMENTS}

\section{A. Method}

In order to experimentally validate the GZ-MSS relationship derived in this study, DDM datasets from the TDS-1 mission were used. For each DDM, metadata include SP position, signal-to-noise ratio (SNR) at the power peak, and incidence
DD domain

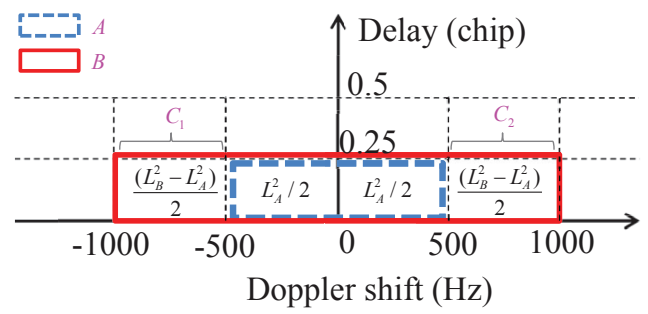

Fig. 3. Determination of spatial area for each DD pixel. DD intervals $A$

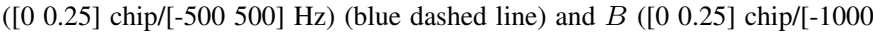
1000] Hz) (red solid line) are shown, corresponding to resolution $L_{A}$ and $L_{B}$, respectively.

angle at SP and collection time. The following pre-processing steps and data quality control were applied: 1) GNSS-R DDMs with SNR greater than $3 \mathrm{~dB}$ (to mitigate the effect of noise), 2) SP latitude between $55^{\circ} \mathrm{S}$ and $55^{\circ} \mathrm{N}$ (to avoid sea ice), 3) SP located in the ocean at a distance of at least $100 \mathrm{~km}$ from the coast (to avoid land contamination), and 4) Only pixels of normalized value greater than a certain threshold (arbitrarily set to 0.2) are retained in this process, to mitigate the effect of noise found in real data. After subtracting the noise floor, each DDM was normalized to its maximum, and incoherently averaged using an adaptive window size (see [19] for details) to reduce the effect of speckle noise.

Collocated in-situ roughness measurements were derived from NDBC buoy data and used as ground-truth. Buoy-derived MSS data were collocated within a radius of $100 \mathrm{~km}$ of the SP location, and a maximum temporal offset of $30 \mathrm{~min}$ from DDM acquisition. Details of the method for MSS determination from buoy data can be found in [28].

\section{B. Data, Results and Discussion}

Three DDM datasets collected by the TDS- 1 satellite near the Gulf of Alaska, Hawaii and Bermuda in 2015 were used. We used the Alaska dataset for algorithm calibration, applied least-squares fitting to the model expressed in Eq. (10) and found $m_{T D S-1}=4.3 \cdot 10^{-8}$, which is in very good agreement with the parameter derived from simulations $\left(m_{T D S-1-S I M}=4.4 \cdot 10^{-8}\right)$.

The Hawaii and Bermuda datasets were reserved and used for validation and assessment of the retrieval performance of the scheme presented; results are shown in Fig. 4. Overall, we found that estimates obtained with our method are in good agreement with ground-truth data, with a correlation coefficient (including calibration and validation datasets) equal to $r=0.73$. Although a generally good agreement is found, some discrepancies can be noticed. The factors potentially affecting the performance of our strategy include: 1) The GZ- 
MSS relationship was derived assuming homogeneous seasurface roughness, 2) the collocation criteria adopted is not particularly tight (100 km/30 min), 3) only DD pixels above a certain power threshold have been taken into account for GZ size evaluation, 4) GZ estimation error can be over $25 \%$ for DD pixels far away from the SP [24], 5) Noise in DDM can also affect the GZ estimation.

We note that surface conditions included in this analysis (MSS $<4 \cdot 10^{-3}$ ) are associated with low winds (with NDBC buoy data reporting wind speed between 1 and $6 \mathrm{~m} / \mathrm{s}$ ). This effect is likely to be attributed to data quality control selecting GNSS-R signals of relatively high SNR, generally associated with lower winds.

It is further noted that the size of the GZ of a satellite GNSS$\mathrm{R}$ system can cover a substantially large area, potentially up to hundreds of kilometers. This introduces an intrinsic limitation in the proposed approach, particularly so in the presence of highly heterogenous surface conditions. Conversely, airborne such as balloon, jet-powered aircraft and U-2 based GNSS-R sensors are characterized by much smaller GZs (of the order of hundreds of meters, depending on altitude) and for that are less affected by this problem. However, airborne GNSS-R data are not readily accessible, and for practical reasons satellite data have been employed in this work as a proof of concept.

\section{CONCLuSions}

The relationship between sea surface roughness (MSS) and area of the glistening zone (GZ) of a GNSS-R system was investigated. First, an analytical formulation of the link between spatial extension of the GZ, incidence angle and surface MSS was derived. Since the GNSS-R satellite measurements from the TDS- 1 mission provide the first reasonably large datasets from much higher altitudes rather than the past earth-based experiments for testing retrieval methods, this model was thus experimentally demonstrated using such data and collocated NDBC buoy-derived surface roughness data. Overall, results show that GNSS-R-retrieved MSS estimates are in good agreement with in-situ data, with an overall correlation coefficient of $r=0.73$. A number of limiting factors were also discussed, including effects linked to data quality control, pre-processing and extension of the GZ of a spaceborne GNSS-R system. Possible future work includes the assessment of this scheme in the context of an airborne GNSS-R system, together with the adoption of more realistic surface models representing also non-Gaussian features and anisotropy of the sea surface.

\section{ACKNOWLEDGMENT}

The authors are grateful to the TDS-1 Team for the work of making the GNSS-R DDM data available, at www.merrbys. co.uk.

\section{REFERENCES}

[1] J. Marchan-Hernandez et al., "Sea-state determination using GNSS-R data," IEEE Geosci. Remote Sens. Lett., vol. 7, no. 4, pp. 621-625, Oct. 2010.

[2] M. P. Clarizia and C. S. Ruf, "Wind speed retrieval algorithm for the Cyclone Global Navigation Satellite System (CYGNSS) mission," IEEE Trans. Geosci. Remote Sens., vol. 54, no. 8, pp. 4419-4432, Aug. 2016.

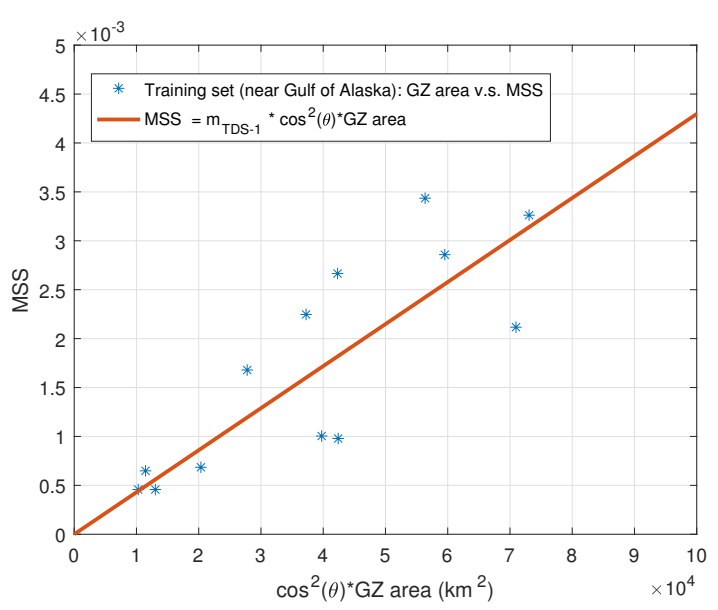

(a)

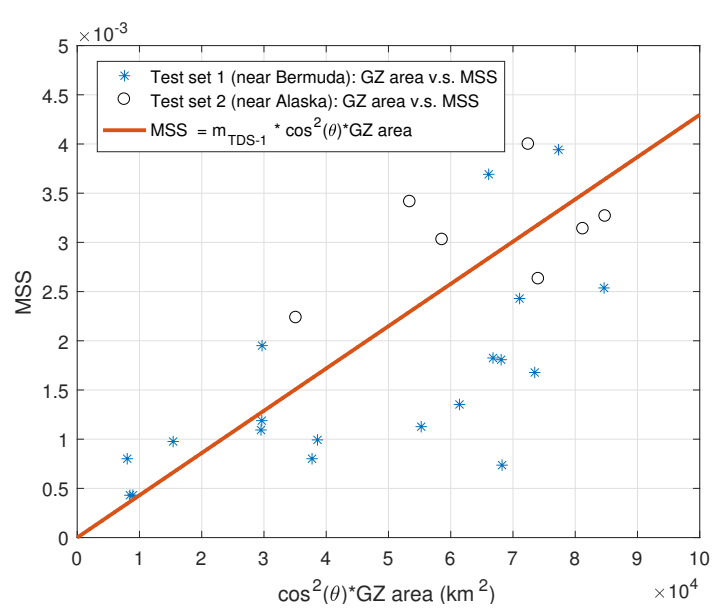

(b)

Fig. 4. Relationship between area of the glistening zone (GZ) and surface roughness (MSS): (a) training dataset (Gulf of Alaska) and (b) validation datasets (Hawaii and Bermuda).

[3] J. F. Marchan-Hernandez et al., "Correction of the sea state impact in the L-band brightness temperature by means of delay-Doppler maps of global navigation satellite signals reflected over the sea surface," IEEE Trans. Geosci. Remote Sens., vol. 46, no. 10, pp. 2914-2923, Oct. 2008.

[4] N. Rodriguez-Alvarez et al., "Airborne GNSS-R wind retrievals using delay-Doppler maps," IEEE Trans. Geosci. Remote Sens., vol. 51, no. 1, pp. 626-641, Jan. 2013.

[5] E. Valencia, V. U. Zavorotny, D. M. Akos, and A. Camps, "Using DDM asymmetry metrics for wind direction retrieval from GPS ocean-scattered signals in airborne experiments," IEEE Trans. Geosci. Remote Sens., vol. 52, no. 7, pp. 3924-3936, Jul. 2014.

[6] G. Foti et al., "Spaceborne GNSS reflectometry for ocean winds: First results from the UK TechDemoSat-1 mission," Geophys. Res. Lett., vol. 42, no. 13, pp. 5435-5441, Jul. 2015.

[7] O. Germain et al., "The Eddy experiment: GNSS-R speculometry for directional sea-roughness retrieval from low altitude aircraft," Geophys. Res. Lett., vol. 31, no. 21, Nov. 2004, Art. no. L12306.

[8] S. Gleason et al., "Detection and processing of bistatically reflected GPS signals from low Earth orbit for the purpose of ocean remote sensing," IEEE Trans. Geosci. Remote Sens., vol. 43 no. 6, pp. 1229-1241, Jun. 2005.

[9] M. P. Clarizia et al., "Analysis of GNSS-R delayDoppler maps from the UK-DMC satellite over the ocean," Geophys. Res. Lett., vol. 36, p. L02608, 2009.

[10] C. Li and W. Huang, "An algorithm for sea surface wind field retrieval from GNSS-R delay-Doppler map," IEEE Geosci. Remote Sens. Lett., vol. 11, no. 12, pp. 2110-2114, Dec. 2014. 
[11] J. Tye, P. Jales, M. Unwin, and C. Underwood, "The first application of stare processing to retrieve mean square slope using the SGR-ReSI GNSSR experiment on TDS-1," IEEE J. Sel. Topics Appl. Earth Observ. Remote Sens., vol. 9, no. 10, pp. 4669-4677, Oct. 2016.

[12] E. Valencia et al., "Ocean surface's scattering coefficient retrieval by delayDoppler map inversion," IEEE Geosci. Remote Sens. Lett., vol. 8. no. 4, pp. 750-754, Jul. 2011.

[13] A. Camps et al., "Sensitivity of GNSS-R spaceborne observations to soil moisture and vegetation," IEEE J. Sel. Topics Appl. Earth Observ. Remote Sens., vol. 9, no. 10, pp. 4730-4742, Oct. 2016.

[14] Y. Jia, P. Savi, D. Canone, and R. Notarpietro, "Estimation of surface characteristics using GNSS LH-reflected signals: Land versus water," IEEE J. Sel. Topics Appl. Earth Observ. Remote Sens., vol. 9, no. 10, pp. 4752-4758, Oct. 2016.

[15] E. Valencia, A. Camps, H. Park, and N. Rodriguez-Alvarez, "Oil slicks detection using GNSS-R," in Proc. IEEE Int. Geosci. Remote Sens. Symp., Vancouver, Canada, Jul. 2011, pp. 4383-4386.

[16] C. Li, W. Huang, and S. Gleason, "Dual antenna space-based GNSS-R ocean surface mapping: Oil slick and tropical cyclone sensing," IEEE J. Sel. Topics Appl. Earth Observ. Remote Sens., vol. 8, no. 1, pp. 425-435, Jan. 2015.

[17] Q. Yan and W. Huang, "GNSS-R delay-Doppler map simulation based on the 2004 Sumatra-Andaman tsunami event," J. Sensors, vol. 2016, p. 2750862, 2016.

[18] Q. Yan and W. Huang, "Tsunami detection and parameter estimation from GNSS-R delay-Doppler map," IEEE J. Sel. Topics Appl. Earth Observ. Remote Sens., vol. 9, no. 10, pp. 4650-4659, Oct. 2016.

[19] Q. Yan and W. Huang, "Spaceborne GNSS-R sea ice detection using delay-Doppler maps: First results from the UK TechDemoSat-1 mission," IEEE J. Sel. Topics Appl. Earth Observ. Remote Sens., vol. 9, no. 10, pp. 4795-4801, Oct. 2016.

[20] A. Alonso-Arroyo, V. U. Zavorotny, and A. Camps, "Sea ice detection using UK TDS-1 GNSS-R data," IEEE Trans. Geosci. Remote Sens., vol. 55, no. 9, pp. 4989-5001, Sep. 2017.

[21] Q. Yan, W. Huang, and C. Moloney, "Neural networks based sea ice detection and concentration retrieval from GNSS-R delay-Doppler maps," IEEE J. Sel. Topics Appl. Earth Observ. Remote Sens., vol. 10, no. 8, pp. 3789-3798, Aug. 2017.

[22] G. A. Hajj and C. Zuffada, "Theoretical description of a bistatic system for ocean altimetry using the GPS signal," Radio Sci., vol. 38, no. 5, pp. 1-10, Oct. 2003.

[23] V. U. Zavorotny and A. G. Voronovich, "Scattering of GPS signals from the ocean with wind remote sensing application," IEEE Trans. Geosci. Remote Sens., vol. 38, no. 2, pp. 951-964, Mar. 2000.

[24] M. P. Clarizia and C. S. Ruf, "On the spatial resolution of GNSS reflectometry," IEEE Geosci. Remote Sens. Lett., vol. 13, no. 8, pp. 10641068, Aug. 2016.

[25] S. J. Katzberg, O. Torres, and G. Ganoe, "Calibration of reflected GPS for tropical storm wind speed retrievals," Geophys. Res. Lett., vol. 33, no. 18, p. L18602, 2006.

[26] S. Gleason, "Remote sensing of ocean, ice and land surfaces using bistatically scattered GNSS signals from low Earth orbit," Ph.D. dissertation, Univ. Surrey, U.K., 2006.

[27] S. Lowe et al., "First spaceborne observation of an earth-reflected GPS signal," Radio Sci., vol. 37, no. 1, pp. 7-1-7-28, Feb. 2002.

[28] T. Elfouhaily, B. Chapron, K. Katsaros, and D. Vandemark, "A unified directional spectrum for long and short wind-driven waves," J. Geophys. Res., vol. 102, no. C7, pp. 15781-15796, Jul. 1997. 